UNIVERSIDADE DE SÃO PAULO

INSTITUTO DE GEOCIÊNCIAS

\title{
RADAR DE PENETRAÇÃO NO SOLO E RESISTIVIDADE ELÉTRICA APLICADOS NAS FORMAÇÕES SÃO SEBASTIÃO E MARIZAL DAS SUB-BACIAS DO TUCANO SUL E CENTRAL, CRETÁCEO (BA).
}

Larissa Natsumi Tamura

Orientador: Prof. Dr. Renato Paes de Almeida

DISSERTAÇÃO DE MESTRADO

Programa de Pós- Graduação em Geoquímica e Geotectônica

SÃO PAULO

2015 
Larissa Natsumi Tamura

\section{RADAR DE PENETRAÇÃO NO SOLO E RESISTIVIDADE ELÉTRICA APLICADOS NAS FORMAÇÕES SÃO SEBASTIÃO E MARIZAL DAS SUB-BACIAS DO TUCANO SUL E CENTRAL, CRETÁCEO (BA).}

Dissertação apresentada ao Instituto de Geociências da Universidade de São Paulo para obtenção de título de Mestre em Geologia.

Área de concentração: Geotectônica

Orientador: Prof. Dr. Renato Paes de Almeida 
Autorizo a reprodução e divulgação total ou parcial deste trabalho, por qualquer meio convencional ou eletrônico, para fins de estudo e pesquisa, desde que citada a fonte.

Ficha catalográfica preparada pelo Serviço de Biblioteca e Documentação do Instituto de Geociências da Universidade de São Paulo

Tamura, Larissa Natsumi

Radar de penetração no solo (GPR) e resistividade elétrica (ER) aplicados nas Formações São Sebastião e Marizal das sub-bacias do Tucano sul e Central, Cretáceo (BA) / Larissa Natsumi Tamura. - São Paulo, 2015

84 p.: il

Dissertação (Mestrado) : IGc/USP

Orient.: Almeida, Renato Paes

1. Radar facies 2. Métodos geofísicos 3. Formação São Sebastião e Marizal 4. Ambiente fluvial I. Título 


\section{AGRADECIMENTOS}

Agradeço imensamente ao meu orientador Prof. Dro Renato Paes de Almeida que me deu oportunidade de iniciar um novo projeto em seu grupo. Ele me incentivou e acreditou nessa nova ideia me dando força e confiança para seguir em frente. Sou muito grata a Liliane Janikian Paes de Almeida que também me auxiliou e me apoiou tanto na pesquisa quanto na comunicação com o Prof. Dro Renato quanto na sua ausência.

Agradeço aos colegas e amigos da pós-graduação, em relação às explicações sobre a geologia, indicações bibliográficas, ajuda na coleta de dados, e auxilio com programas de processamento. Sempre que precisei pude contar a ajuda de vocês: André Marconato, André Stern, Bernardo Tavares Freitas, Carlos Carrasco, Cristiano Galeazzi Padalino, Felipe Torre Figueiredo, Mariana Cossa de Oliveira, Marta Jácomo, Selma Rodrigues, Samuel Abdala e Simone Carrera.

Aos docentes que sempre estiveram dispostos a me ensinar e me ajudar nessa nova etapa, agradeço imensamente o apoio de vocês: Assis Cavarallo, Fábio Taioli, José Faraco Gallas e Milene Fornati. Também sou muito grata aos técnicos que me ensinaram e auxiliaram na execução em campo: Fernando Augusto Saraiva (pesquisador), Ernande Costa e ao Samuca.

Agradeço a Fundação de Amparo à Pesquisa do Estado de São Paulo (FAPESP) pelo auxílio financeiro de pesquisa (Processo № 2013/01825) e ao programa de pós graduação de Geotectônica e Geoquímica CAPES PROEX (Processo № 558/2011). Também agradeço ao Conselho Nacional de Desenvolvimento Científico e Tecnológico pela bolsa concedida (Processo № 832194/1999-9) por meio demanda de cota institucional.

E finalmente agradecer a minha família, namorado e amigos que sempre me apoiaram na decisão de fazer o mestrado, que proporcionaram alegria e tranquilidade em dias difíceis, e torceram muito para que esse dia chegasse. 


\section{RESUMO}

Um dos motivos para o avanço em caracterização de estrutura interna em depósitos fluviais é a utilização de métodos geofísicos. Um método amplamente aplicado em ambientes fluviais é o Radar de Penetração no Solo (GPR - Ground Penetrating Radar). Por meio dele é possível fazer interpretações por radar fácies, frequentemente aplicadas em rios ativos, porém em depósitos antigos esse método não é amplamente testado. Em contra partida, há um grande interesse nesse tipo de estudo devido à importância de modelos de reservatórios análogos de hidrocarbonetos.

Os afloramentos das Formações São Sebastião e Marizal da Bacia do Tucano foram amplamente estudados em relação à sedimentologia por meio de estudos de análises de fácies e arquitetura deposicional. Porém, o estudo arquitetural com métodos geofísicos ainda não havia sido executado nessa área. Desta forma, a principal contribuição do presente trabalho foi aplicar métodos geofísicos em afloramentos bem expostos e comparar os resultados com estudos sedimentares feitos na área e outros trabalhos feitos em rios ativos.

Foram utilizados dois métodos. O primeiro método teve como finalidade aprimorar as ferramentas de interpretação de radar fácies por meio de comparações de dados GPR com estruturas sedimentares e elementos arquiteturais, considerando a resolução do método e suas limitações. Para complementar o estudo foi introduzido um segundo método, denominado resistividade elétrica (ER - eletric resistivity), que contribui nas investigações litológicas em subsuperfície e auxilia o método GPR em camadas muito condutivas.

Através dos resultados obtidos pelo método GPR foi possível identificar oito radar fácies diferentes: 1 ) Refletores levemente ondulados com truncamentos de baixo ângulo. Foram interpretados como estratos ondulados, cruzados de baixo ângulo e limites de série sub-horizontais. 2) Refletores inclinados com grande amplitude e dentro deles refletores de menor amplitude inclinados. Foram interpretados como limites de séries inclinados separando conjuntos de estratos cruzados tabulares. 3) Refletores inclinados com grande amplitude e dentro deles refletores de menor amplitude, descontínuos e de forma de côncava para o topo. Foram interpretados como limites de séries inclinados separando conjuntos de estratos cruzados 
acanalados. 4) Refletores inclinados com alto ângulo em conjuntos métricos, passando lateralmente para as radar fáceis 2 ou 3. Os refletores inclinados são interpretados como resultado de estratos cruzados de grande porte. 5) Refletores com forma levemente ondulada ou côncava para o topo sendo recobertos por refletores horizontais com terminações em onlap. Foram interpretados como estruturas de corte e preenchimento com forma canalizada e preenchimento interno plano-paralelo ou com séries de menores que a resolução do método. 6) Refletores sub-horizontais de grande amplitude e dentro deles refletores de forma côncava para o topo com menor amplitude. Foram interpretados como limites de séries sub-horizontais separando conjuntos de estratos cruzados acanalados. 7) Refletores horizontais contínuos bem marcados e dentro deles refletores inclinados de menor amplitude. Foram interpretados como limites de séries horizontais e estratos internos cruzados tabulares. 8) Refletores com grande amplitude, inclinados em alto ângulo. Foram interpretados como estratos cruzados de grande porte de dunas eólicas.

Ao comparar as seções de GPR com as estruturas sedimentares do afloramento observou-se que houve compatibilidade entre os refletores encontrados nas seções GPR e as estruturas sedimentares observadas em afloramento. Em alguns casos nota-se que a resolução do método é eficaz para identificar estruturas decimétricas, dificilmente encontradas em trabalhos de radar fácies. Porém, as profundidades das seções não ultrapassaram de $5 \mathrm{~m}$, ou seja, foi possível identificar refletores em alta resolução com uma antena de $100 \mathrm{MHz}$, mas em subsuperfície rasa.

Os perfis de ER conseguiram diferenciar as camadas arenosas das peliticas, o que pode justificar o motivo pelo qual alguns locais as seções GPR não obtiveram resultados relevantes.

Desta forma, os métodos geofísicos aplicados mostraram grande potencial para estudos futuros sobre a arquitetura deposicional das unidades investigadas, levando a resultados comparáveis aos estudos de afloramentos e com resolução maior que a normalmente obtida em sucessões inconsolidadas. 


\section{ABSTRACT}

One of the factors for the advances in the characterization of fluvial deposits internal structures is the application of geophysical methods. A frequently applied method in fluvial environments is the Ground Penetrating Radar. Through this method, it is possible to interpret radar facies, often focusing on active river systems, nevertheless the method is not yet extensively tested in the ancient rock record. On the other hand, there is great interest in this type of study due to the importance of hydrocarbon reservoir analogue models.

The São Sebastião Formation and Marizal Formation outcrops in the Tucano Basin have been subject to facies and architectural element analysis in previous works. Nevertheless, the architectural study through geophysical methods has not been applied in the area. Therefore, the main goal of the present work was to apply geophysical methods to well exposed outcrops and to compare the results with those of results from similar surveys developed in active fluvial systems.

Two basic methods were applied. The first method with the aim of contributing to the improvement of the interpretation tools of radar facies through the comparison of GRP data with sedimentary structures and architectural elements, considering the method resolution and limitations. In order to fill in the gaps, the second method, named electrical resistivity (ER), contributed to the lithological investigation in subsurface, imaging very conductive layers.

Through the results obtained in the GPR surveys it was possible to identify eight radar facies: 1) Slightly undulated reflector with low-angle truncations. These were interpreted as undulated strata, low angle cross stratification and irregular set boundaries with cross set bellow the detection limit of the surveys. 2) High amplitude inclined reflectors, bounding lower amplitude reflectors inclined at greater angles. These were interpreted as inclined cross set boundaries with internal planar cross sets. 3) High amplitude inclined reflectors, bounding lower amplitude, concave-up, discontinuous reflectors. These were interpreted as inclined cross set boundaries with internal through cross sets. 4) High angle inclined reflectors disposed in metric sets passing laterally into 2 or 3 . These were interpreted as large-scale planar cross-sets. 5) Slightly undulated concave-up reflectors covered by horizontal reflectors with onlap 
terminations. Interpreted as cut and fill structures filled by planar horizontal stratified sediment or cross set smaller than survey resolution. 6) Sub-horizontal, high amplitude reflectors with internal lower amplitude concave up discontinuous reflectors. Interpreted as trough cross sets with by sub-horizontal set boundaries. 7) Well defined continuous horizontal reflectors with internal lower amplitude inclined reflectors. Interpreted as horizontal set boundaries with internal planar cross sets. 8) Meter-scale high amplitude high angle inclined reflectors. Interpreted large-scale aeolian dune foresets.

Comparison between the GPR sections and the described sedimentary structures revealed compatibility between the reflector geometries and the sedimentary structures. In some situations, the resolution enabled the recognition of decimetric structures, seldom described in published radar facies surveys. On the other hand, depth of investigation did not surpass $5 \mathrm{~m}$. In this way, using a $100 \mathrm{~Hz}$ antenna, it was possible to identify reflectors with great resolution, but only in the shallow subsurface.

ER profiles enabled the recognition of sand and clay rich beds, bringing light into the question of why some GPR surveys did not lead to good results.

Therefore, the applied geophysical methods revealed a great potential for further works on the depositional architecture of the studied units, leading to results comparable with those obtained through the direct study of outcrops, and with a greater resolution than the commonly obtained in unlithified successions. 


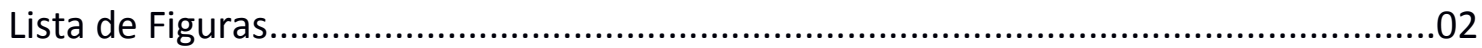

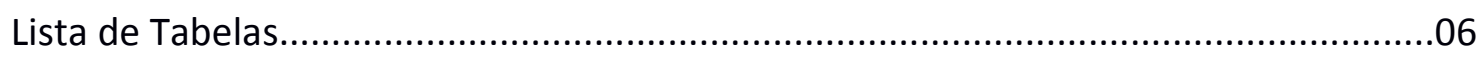

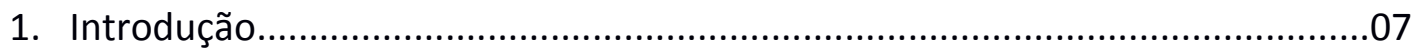

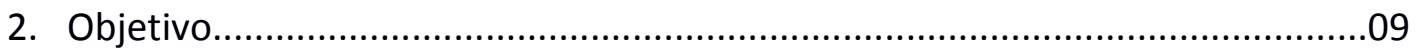

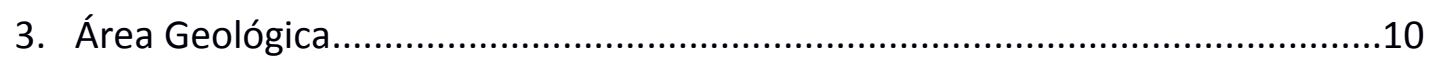

3.1 Bacias Recôncavo-Tucano-Jatobá...........................................................10

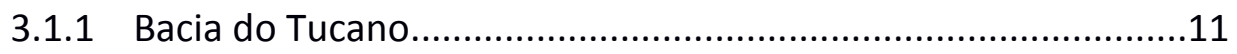

3.1.1.1 Formação São Sebastião............................................15

3.1.1.2 Formação Marizal......................................................16

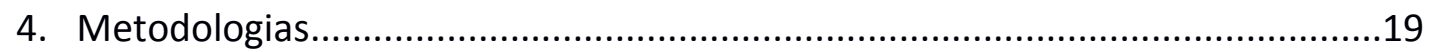

4.1 Radar de penetração no solo..................................................................19

4.1.1 Fundamentos Teóricos...............................................................20

4.1.2 Princípios do método................................................................24

4.1.3 Relação entre resolução, frequência e profundidade......................25

4.1.4 GPR em sedimentos.................................................................28

4.1.5 Aquisição e processamento de dados...........................................30

4.1.6 Trabalhos anteriores sobre GPR em ambientes fluviais.................32

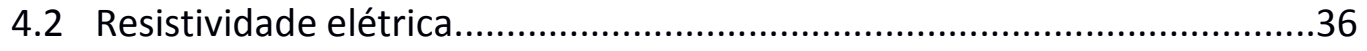

4.2.1 Fundamentos Teóricos ...............................................36

4.2.2 Princípios da técnica de investigação..............................38

4.2.3 Propriedades elétricas em rochas e minerais..................40

4.2.4 Aquisição e processamento de dados.............................41

4.2.5 Trabalhos anteriores com o uso de ER com GPR ............42

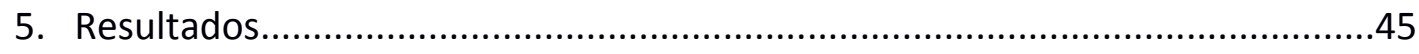

5.1 Radar de Penetração de solo............................................................45

5.1.1 Comparação das seções GPR com afloramentos ......................59

Resistividade elétrica...........................................................67

5.2.1 Comparação com fácies sedimentares e geometrias em escala de

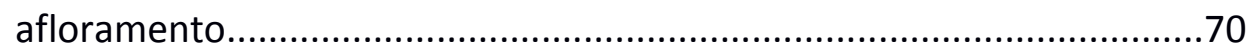

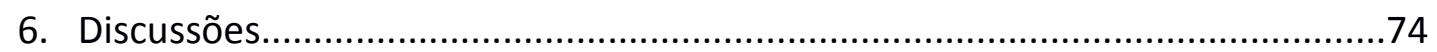

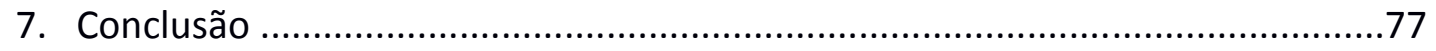

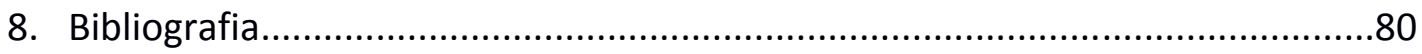




\section{SUMÁRIO DE FIGURAS}

Figura 1 - A) Localização geográfica das bacias R-T-J. B) Esboço geológico das bacias Recôncavo, Tucano (Norte, Central e Sul) e Jatobá (Magnavita et al., 2003) ...10

Figura 2 - Limites e arcabouço estrutural da Bacia do Tucano (Magnavita et al., 2003)

Figura 3 - Mapa regional de anomalia Bouger mostrando o rift R-T-J, com destaque no Baixo de Cícero Dantas (Santos et al., 2010)

Figura 4 - Carta estratigráfica e aspectos tectono-deposicionais da Bacia do Tucano (Santos et al., 2010)

Figura 5 - Coluna estratigráfica da Formação de São Sebastião (Santos et al., 2010)

Figura 6 - Coluna estratigráfica da Formação de Marizal (Santos et al., 2010)

Figura 7 - a) aquisição dos dados em um ponto do levantamento e b) resultado do perfil da reflexão do radar (Neal, 2004) .25

Figura 8 - Dois métodos de aquisição mais comuns utilizados em levantamentos GPR: A) Common of set e B) Common Mid Ponit (Neal, 2004) 25

Figura 9 - A) Ondas eletromagnéticas propagando-se em uma superfície em forma de cone, ilustrando a zona de máxima resolução vertical. B) Ondas mais próximas ao centro da zona de Fresnel contribuem para uma maior amplitude. C) A largura da zona de Fresnel está em função da profundidade do refletor e da frequência (Neal, 2004) .27

Figura 10 - Terminologia para definir e descrever radar fácies. A) Geometria da reflexão, B) Forma 3-D externa dos pacotes de radar e C) Fácies detectadas pelo radar (Neal, 2004) 30

Figura 11 - Levantamento GPR com antena de $100 \mathrm{MHz}$ 31

Figura 12 - Esquema com quatro eletrodos de como a corrente circula e onde o potencial de forma (Braga, 2007) .38

Figura 13 - Esquema do arranjo dipolo-dipolo para vários níveis de investigação........39

Figura 14 - Configuração do levantamento ER .42

Figura 15 - A) Mapa geológico Bacia do Tucano adaptado de Freitas (2014) com os pontos de onde foram feitos os levantamentos. B) Mapa das principais cidades e estradas da área de estudo (Freitas, 2014)

Figura 16 - Levantamento GPR no Ponto 1 do mapa da Figura 15, a seção mostra em alguns trechos refletores levemente ondulados contínuos, interpretados estratos ondulados, cruzados de baixo ângulo e limites de série subhorizontais. .51

Figura 17 - Seção de GPR no Ponto 2, notam-se os refletores em forma côncava para cima e dentro deles refletores plano-paralelos com terminação em onlap. Foram interpretados como estruturas de corte e preenchimento com forma canalizada 
e o preenchimento interno de arenito ou pelito plano-paralelo ou com séries de cruzadas menores que a resolução do método

Figura 18 - No Ponto 3 (Figura 15) a seção A mostra no início da seção refletores inclinados com alta amplitude e dentro de cada um deles refletores de menor amplitude de forma côncava para cima e refletores também de menor amplitude cruzados tabulares. Interpretados como limites de séries cruzados de dunas cavalgantes e estratos internos cruzados acanalados ou estratos internos cruzados tabulares representando as frentes de cada duna preservada. Mais adiante se observa um refletor com uma forte amplitude e inclinado, interpretado como segregação de sedimentos e no final da seção também ocorre outra segregação sedimentar. .52

Figura 19 - Na seção B do Ponto 3 (Figura 15) nota-se no meio da seção a presença de refletores inclinados com alta angulosidade representando os estratos frontais e nas laterais da seção observa-se refletores subparalelos contínuos e dentro deles refletores inclinados de menor amplitude, interpretados como limites de série subparalelos de dunas cavalgantes em corte ortogonal à direção de migração com estratos internos cruzados tabulares que são as frentes de dunas preservadas

Figura 20 - A última seção (C) do Ponto 3 na maior parte da seção é representada por refletores contínuos levemente ondulados e em alguns locais dentro desses refletores conseguem-se notar alguns refletores de menor amplitude com forma côncava para cima, interpretados como limites de séries de estratos cruzados acanalados formados por dunas cavalgantes com crista sinuosa em corte ortogonal à direção de migração com estratos internos cruzados acanalados, que são as frentes de dunas preservadas .53

Figura 21 - A seção de GPR do Ponto 6 (Figura 15) apresenta refletores com alta amplitude levemente ondulados e contínuos, dentro deles em alguns locais observa-se a existência de refletores de baixa amplitude em forma côncava para cima, essa seção foi interpretada como limites de séries de dunas cavalgantes com crista sinuosa em corte ortogonal à direção de migração com estratos internos cruzados acanalados que são as frentes de dunas preservadas. .53

Figura 22 - Ponto 5 a seção GPR foi feita em ambiente sedimentar eólico, nota-se uma penetração um pouco maior que nas seções anteriores. Em quase toda seção apresentam-se refletores de alta amplitude e inclinados em direção sudoeste representando estratos cruzados de grande porte formados por dunas eólicas e os refletores que truncam os estratos cruzados sugerem superfícies inferiores erosivas para sucessões de interdunas .54

Figura 23 - Seção GPR do Ponto 1 com uma foto do afloramento mostrando cruzadas de baixo ângulo que aparecem como refletores ondulados com truncamentos de baixo ângulo na seção de GPR. Feições menores, como marcas onduladas e laminações cruzadas, estão abaixo da resolução do levantamento. .62

Figura 24 - A) Seção GPR do Ponto 2 com a ampliação de dois canais principais e a foto de cada um desses canais ao lado, mostrando o truncamentos entre refletores e 
terminações em onlap contra refletores côncavos para o topo com comprimento métrico, foram interpretados como preenchimento de feições canalizadas, compatíveis com o observado no afloramento, que apresenta canais preenchidos por arenitos finos laminados e arenitos finos com estratificação cruzada acanalada em séries decimétricas. B) Parte do afloramento descrito por Figueiredo (2013) mostrando a localização de ambos os canais no afloramento.. .63

Figura 25 - O levantamento GPR no Ponto 4 na seção A. Foram encontradas basicamente quatro mudanças nos padrões dos refletores e as fotos de partes do aforamento ilustram essas mudanças nas estruturas sedimentares. Quadro azul- arenitos médios com raros grânulos esparsos, organizados em séries de cruzadas acanaladas com limites de séries inclinados em direção ao levantamento da seção GPR. Quadro laranja- arenitos grossos a médios, com grânulos pequenos, seixos esparsos e forte segregação granulométrica, com níveis grossos maciços em meio aos arenitos médios. Os limites de séries tem pelo menos $2 \mathrm{~m}$ de espessura com cruzada tabular marcada por forte segregação granulométrica, mais ortogonal ao levantamento. Quadro verde- mesmas séries são de grande porte, tabulares com segregação de arenito muito grosso rico em pequenos seixos nos estratos cruzados. Quadro amarelo - arenitos muito grossos com segregação e espessura de série de mais de $1,5 \mathrm{~m}$ .64

Figura 26 - A seção GPR foi passada exatamente na superfície sobre a qual encontramse as pessoas na Figura da direita. $O$ afloramento logo abaixo do levantamento revela arenitos médios a grossos com séries de estratificação cruzada tabular separadas por limites inclinados. Uma superfície lateralmente contínua, posicionada menos de $1 \mathrm{~m}$ abaixo do topo da seção, separa um conjunto de séries inclinadas a ângulos mais altos de outro com limites de série de baixo ângulo. Na seção de GPR essa superfície é claramente representada por um refletor contínuo suavemente côncavo para o topo, sobre o qual os refletores superiores terminam em onlap. A maior parte dos refletores representa limites de séries e alguns refletores de menor amplitude associados a estratos cruzados internos podem ser reconhecidos.

Figura 27 - Na seção de GPR, a estratificação cruzada de grande porte com superfícies internas de reativação é claramente visível. A maior penetração do sinal obtida nesta seção permitiu o reconhecimento de estratos cruzados de até $5 \mathrm{~m}$ de espessura. Refletores sub-horizontais associam-se aos arenitos com laminação plano-paralela, e suas terminações em onlapsobre refletores que truncam os estratos cruzados sugerem superfícies inferiores erosivas para as sucessões de interdunas

Figura 28 - Seção modelada do Ponto 2. A parte mais rasa da seção é mais resistiva sugerindo presença de arenitos, em maior profundidade a condutividade aumenta interpretando-se como uma espessa camada pelítica e mais abaixo uma camada mais resistiva indicando novamente uma mudança litológica......69

Figura 29: Seção modelada do Ponto 3. Nota-se que entre no início da a presença de uma camada pelítica. No meio da seção há uma grande anomalia resistiva, 
indicando a presença de arenitos limpos mais ao topo da seção. No final da seção mais superficialmente, condutividade aumenta sugerindo um material mais pelítico, em camada sobreposta aos arenitos. No final da seção observam-se duas anomalias mais resistivas que significa a presença de arenitos arcóseanos. 69

Figura 30 - Seção do levantamento ER no Ponto 2 com fotos de cada tipo de rocha sedimentar apenas da parte mais resistiva do topo (arenitos finos micáceos e siltosos com laminação cruzada cavalgante, por vezes preservando marcas onduladas (foto do meio), arenitos finos com laminação plano-paralela (foto à direita), arenitos finos a médios com estratificação cruzada acanalada (foto à esquerda). Em maior profundidade há exemplos de estruturas sedimentares não aflorantes....

Figura 31 - A) Parte da foto do afloramento do Ponto 3 adaptado de Figueiredo et al.,2013. B) Seção modelada do levantamento ER.O padrão de variação de camadas mais e menos resistivas no levantamento ER coincide em alguns locais com a intercalação métrica entre sucessões de arenitos conglomeráticos e arenitos finos siltosos com níveis pelíticos descritas por Figueiredo (2013) 


\section{SUMÁRIO DE TABELAS}

Tabela 1: Definições e unidades utilizadas no método GPR............................................20

Tabela 2: Relação entre a frequência da antena e profundidade de penetração (Porsani,

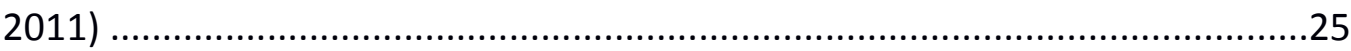

Tabela 3: Valores de resolução teórica GPR em ambientes sedimentares típicos para diferentes frequências de antenas (Jol \& Bristow, 2003) . .25

Tabela 4: Valores das frequências das antenas e a resolução vertical para cada uma delas (Porsani, 2011) .26

Tabela 5: Exemplos de alguns materiais geológicos e suas propriedades elétricas (Neal,2004) .28

Tabela 6: Definições e unidades utilizadas no método ER .35

Tabela 7: Valores de resistividade adaptado de Gallas (2000) 39

Tabela 8: Radar facies encontradas nas seções acima com descrição e interpretação para cada uma delas. 


\section{CONCLUSÕES}

Os afloramentos das Formações São Sebastião e Marizal da Bacia do Tucano foram amplamente estudados em relação à sedimentologia por meio de estudos de análises de fácies e arquitetura deposicional. Porém, o estudo arquitetural com métodos geofísicos ainda não havia sido executado nessa área. Desta forma, a principal contribuição do presente trabalho foi aplicar métodos geofísicos em afloramentos bem expostos e comparar os resultados com estudos sedimentares feitos na área e outros trabalhos feitos em rios ativos.

Por meio de estudos dos perfis de GPR nas Sub-bacias do Tucano Sul e Central, nas Formações São Sebastião e Marizal foram encontrados oito radar fácies. As radar fácies 2 e 7, 3 e 6 possuem a mesma interpretação de ambiente deposicional, o que as definiram como radar fácies diferentes foi a mudança de inclinação dos refletores na seção GPR e isso dependeu da orientação que em que os levantamentos foram executados nos afloramentos.

1. Refletores levemente ondulados com truncamentos de baixo ângulo foram interpretados como estratos ondulados, cruzadas de baixo ângulo e limites de série sub-horizontais de estratos cruzados abaixo da resolução do levantamento.

2. Refletores inclinados com grande amplitude e dentro deles refletores de menor amplitude inclinados a ângulos mais altos foram interpretados como limites de séries inclinados separando conjuntos de estratos cruzados tabulares. A inclinação dos limites de série na mesma direção dos estratos cruzados sugere migração de dunas na porção frontal de uma barra, com acréscimo para jusante.

3. Refletores inclinados com grande amplitude e dentro deles refletores de menor amplitude, descontínuos e de forma de côncava para o topo foram interpretados como limites de séries separando conjuntos de estratos cruzados acanalados. A inclinação dos limites de série na mesma direção dos estratos cruzados sugere migração de dunas.

4. Refletores inclinados com alto ângulo em séries de espessura métrica e espaçamento decimétrico entre os refletores de maior amplitude. Os refletores inclinados são interpretados como resultado de estratos cruzados de grande porte por migração de barras unitárias. $\mathrm{O}$ alto ângulo e a espessura dos conjuntos de estratos cruzados (mais de $1 \mathrm{~m}$ ) são compatíveis com faces de avalanche em barras unitárias. 
5. Refletores em forma levemente ondulada ou côncava para o topo sendo recobertos por refletores horizontais com terminações em onlap foram interpretados como estruturas de corte e preenchimento com forma canalizada e preenchimento interno plano-paralelo ou com séries de menores que a resolução do método. São interpretados como pequenos canais abandonados, preenchidos por areia ou pelitos em contexto de topos de barras fluviais.

6. Refletores sub-horizontais de grande amplitude e dentro deles refletores de forma côncava para o topo com menor amplitude foram interpretados como limites de séries sub-horizontais separando conjuntos de estratos cruzados acanalados. Ela possui crista levemente sinuosa, em corte ortogonal à direção de migração.

7. Refletores bem marcados plano-paralelos e dentro deles refletores de menor amplitude foram interpretados como limites de séries plano-paralelos de dunas cavalgantes em corte ortogonal à direção de migração e estratos internos cruzados tabulares.

8. Refletores com grande amplitude, inclinados em alto ângulo e refletores subhorizontais com terminações onlap e espaçamento entre eles decimétricos foram interpretados como estratos cruzados de grande porte de dunas eólicas. Os estratos sub-horizontais associam-se aos arenitos com laminação plano-paralela, e com terminações onlap sobre os refletores que truncam os estratos cruzados sugerem superfícies inferiores erosivas para sucessões de interdunas.

Ao comprar as seções de GPR com as estruturas sedimentares do afloramento conclui-se que houve compatibilidade entre os refletores encontrados nas seções GPR e as estruturas sedimentares observadas diretamente. Em alguns casos nota-se que a resolução do método é eficaz para identificar estruturas decimétricos, dificilmente encontradas em trabalhos de radar fácies (e.g. Best et al., 2003 e Smith et al., 2009). Porém, as profundidades das seções não ultrapassaram de $5 \mathrm{~m}$. Ou seja, com uma antena de $100 \mathrm{MHz}$ foi possível identificar refletores em alta resolução, mas em subsuperfície rasa.

Os resultados dos perfis de ER também foram relevantes, visto que o método conseguiu identificar camadas com litologias e formas geométricas diferentes, sendo eficaz principalmente para diferenciar as camadas arenosas das pelíticas. Isso foi muito 
importante pois pode justificar porque em locais onde há camadas pelíticas (mais condutivas) as seções GPR não obtiveram resultados relevantes, justificando a baixa penetração sinal eletromagnético. Dessa maneira, conclui-se que o uso em conjunto do GPR com o ER é uma boa maneira de executar estudos arquiteturais na área de estudo, pois os métodos se complementam. O GPR consegue visualizar perfis em alta resolução, mas limita-se pelo fato de não penetrar em camadas argilosas. Já o ER consegue distinguir diferentes tipos de litologia, identificando camadas mais condutivas (pelíticas) e isso auxilia nos estudos de arquitetura deposicional.

Desta forma, os métodos geofísicos aplicados mostraram grande potencial para mais estudos futuros, devido aos resultados satisfatórios em comparação com os estudos arquiteturais já realizados na área da Sub- Bacia Tucano Sul e Central (Fm. São Sebastião e Marizal) e nos outros estudos de GPR e ER em sedimentos inconsolidados.

Como os resultados foram satisfatórios futuramente seria interessante uma investigação dos métodos em $3 \mathrm{D}$ para conseguir determinar a continuidade dos corpos sedimentares onde não há mais a exposição lateral do afloramento e iniciar estudos arquiteturais desses afloramentos como análogos de reservatórios de hidrocarbonetos. 


\section{REFERÊNCIAS}

Best J.L., Ashworth P. J., Bristow C. S., Roden J. 2003. Three-dimensional sedimentary architecture of large, mid-channel sand braid bar, Jamuna River, Bangladesh. Jornal Sedimentary Research, 73(4): 516-530.

Smith G. H. S., Ashworth P. J., Best J. L., Orfeo O., Parsons D. R. 2009. The sedimentary and alluvial architecture of large braid bar, Rio Paraná, Argentina. Jornal Sedimentary Research, 79: 629-642. 